\title{
Inducement by directional fields of rotational and translational phase ordering in polymer liquid-crystals
}

\author{
A. AISunaidi, ${ }^{1, a)}$ W. K. den Otter, ${ }^{2, b)}$ and J. H. R. Clarke ${ }^{3, c)}$ \\ ${ }^{1}$ Department of Physics and Center of Excellence in Nanotechnology, King Fahd University of Petroleum \\ and Minerals, Dhahran 31261, Saudi Arabia \\ ${ }^{2}$ Faculty of Science and Technology, Faculty of Engineering Technology, and MESA+ Institute \\ for Nanotechnology, University of Twente, P.O. Box 217, 7500 AE Enschede, The Netherlands \\ ${ }^{3}$ School of Chemistry, University of Manchester, P.O. Box 88, Manchester M60 1QD, United Kingdom
}

(Received 10 June 2012; accepted 14 March 2013; published online 19 April 2013)

\begin{abstract}
The effects of aligning fields on models of polymer liquid crystals were simulated using the dissipative particle dynamics method. Exposing a liquid crystal of rod-like particles to a directional field causes a stabilization of the phases with orientational order, shifts the isotropic-nematic and nematic-smectic-A phase transitions to higher temperatures, makes the transitions continuous beyond a critical field strength, and induces weak para-nematic alignment in the zero-field isotropic phase. The interplay of liquid-crystalline ordering, microphase separation, and an alignment field endows the diblock and triblock copolymers studied here with rich phase behavior. The simulations suggest that field-induced orientational ordering can give rise to positional ordering. Reversely, positional ordering resulting from rod-coil demixing may be accompanied by orientational ordering, which is enhanced by external fields. For highly asymmetric rod-coil copolymers, the microphase separation pattern formed by the rigid segments can be altered by an aligning field. (c) 2013 American Institute of Physics. [http://dx.doi.org/10.1063/1.4798462]
\end{abstract}

\section{INTRODUCTION}

The operation of optoelectronic devices based on liquid crystal molecules, such as flat panel displays, light shutters, and optical switches, is governed by the interaction of these rod-like molecules with externally applied fields. Electric and magnetic fields exert torques on the nematogens to induce rotational alignment of the molecules along the field direction. This orientational coupling with an external field has been reported to shift the phase diagrams of these materials, by inducing directional as well as spatial ordering. For example, the application of a DC voltage to a sample of $p$-ethoxybenzylidene- $p^{\prime}$-aminobenzonitrile was reported to cause a shift in the clearing point, i.e., the isotropic-nematic transition, toward higher temperatures. ${ }^{1}$ Similar observations were reported for other types of liquid-crystal molecules like cyanobiphenyl, ${ }^{2} 4^{\prime}$ - $n$-pentyl-4-cyanobiphenyl (5CB), ${ }^{3}$ and stilbene-type liquid crystals. ${ }^{4}$ Lelidis and Durand ${ }^{5}$ investigated a mixture of $4^{\prime}-n$-octyl-4-cyanobiphenyl (8CB) and $4^{\prime}$ $n$-decyl-4-cyanobiphenyl (10CB) which in the absence of an external field only shows a spontaneous isotropic-smectic A transition. Applying weak electric fields induces a weak directional alignment, i.e., para-nematic ordering, while strong enough fields cause a first order para-nematic-nematic transition. This induction of a nematic phase, in a system otherwise showing only isotropic and smectic phases, was predicted theoretically by Rosenblatt. ${ }^{6}$ Isotropic-nematic transitions induced by magnetic fields have been reported for other

\footnotetext{
a)Email: asunaidi@kfupm.edu.sa

b) Email: w.k.denotter@utwente.nl

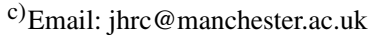

systems such as bent-core liquid-crystal molecules ${ }^{7}$ and colloidal suspensions of monodisperse $f d$ viruses. $^{8}$

Moreover, it was predicted theoretically and confirmed experimentally that strong external fields can introduce critical or tricritical points in the phase diagram beyond which a discontinuous first-order transition vanishes or becomes a second order continuous transition, respectively. To give a few examples, the effect of a magnetic field on the isotropic-nematic phase transition was studied by Wojtowicz and Sheng, ${ }^{9}$ using the Maier-Saupe model, who found that the field-temperature phase diagram shows a critical point similar to the liquid-gas critical point. Khokhlov and Semenov ${ }^{10}$ used the Onsager theory to show that applying a strong disorienting field of the quadrupole type on solutions of rods and solutions of semiflexible chains induces a tricritical point, with the director of the nematic phase being perpendicular to the field direction. This effect of the quadrupole field was also observed by Wensink and Vroege, ${ }^{11}$ who used an Onsager-Parsons density-functional theory to study the effect of an external magnetic field on the nematic order of charged spherocylinders with negative diamagnetic susceptibility (representing Goethite nanorods). Several other authors reported on the critical behavior near the isotropic-nematic and the nematic-smectic A transitions under the effect of fields within the framework of the Landaude Gennes ${ }^{12-14}$ and McMillan theories..$^{15,16}$ Experimentally, Nicastro and Keyes ${ }^{17}$ studied the electric field-induced critical phenomena at the isotropic-nematic transition of MBBA $(N-$ ( $p$-methoxybenzylidene)-p-ylaniline) with negative dielectric constant and 4'- $n$-heptyl-4-biphenyl (7CB) with positive dielectric constant. Lelidis and Durand ${ }^{3}$ studied experimentally the effect of an electric field on the isotropic-nematic 
transition of $5 \mathrm{CB}$. Above a critical field $E_{\mathrm{c}}=140 \mathrm{kV} / \mathrm{cm}$, the transition became a continuous transition. This data were confirmed by Basappa and Madhusudana, ${ }^{2}$ who for octyloxy cyanobiphenyl (8OCB) found a less clear critical point at $E_{\mathrm{c}} \approx 160 \mathrm{kV} / \mathrm{cm}$. Dhara and Madhusudana ${ }^{18}$ have shown experimentally that the isotropic-nematic transition of $4^{\prime}$-butyl4-heptyl-bicyclohexyl-4-carbonitrile (CCN47) with negative dielectric anisotropy becomes continuous beyond a tricritical field estimated to be $170 \mathrm{kV} / \mathrm{cm}$. For the nematic-smectic A transition, Lelidis ${ }^{19}$ estimated the tricritical field for $8 \mathrm{CB}$ to be about $20 \mathrm{kV} / \mathrm{cm}$, corresponding to about $130 \mathrm{~T}$, which is consistent with Yethiraj's ${ }^{20}$ experimental predictions. We refer the reader to Refs. 13, 21, and 22 for reviews on the effect of external fields on the isotropic-nematic and nematicsmectic A transitions.

External fields also affect the microphase separation of polymer liquid crystals, composed of rigid and flexible blocks (rod-coil copolymers). Small-angle X-ray scattering experiments have shown ${ }^{23}$ that the order-disorder temperature in rod-coil copolymers (poly(2,5-di(20-ethylhexyloxy)1,4-phenylenevinylene)-b-polyisoprene) increases due to a stabilization of the smectic phase in the presence of magnetic fields. Also, the alignment of the microdomains resulting from phase separation of block copolymers containing mesogenic blocks can be controlled using external fields. Tao et al. ${ }^{24}$ demonstrated that the rod's director of the rod-coil block copolymers, poly-(2,5-di(2'-ethylhexyloxy)1,4-phenylenevinylene-block-1,4-isoprene) reorients along a 9.4 $\mathrm{T}$ magnetic field through coupling of the magnetic field to the diamagnetic moment of individual rod blocks. This led to alignment of the interfaces perpendicular to the field lines and long range order of a $10 \mathrm{~nm}$ lengthscale. Gopinadhan et al. ${ }^{25,26}$ have reported the controlled rotation of lamellar and cylindrical microdomains of in poly(ethylene oxide-b-6-(40cyanobiphenyl-4-yloxy) hexyl methacrylate) block copolymer using a $6 \mathrm{~T}$ magnetic field. For the lamellar layers, the interfaces align along the applied field while the smectic layers are perpendicular to the field. The cylindrical domains were rotated from perpendicular to parallel orientation with respect to the field. Hamley et al. ${ }^{27}$ have also shown that the morphologies of a series of side-group liquid-crystal block copolymers comprising liquid-crystal (LC) cylindrical domains in a polystyrene matrix could be oriented by slow cooling through the clearing temperature, in the presence of a $1.8 \mathrm{~T}$ magnetic field. Ohta et $_{\text {al }}{ }^{28,29}$ solved a two-dimensional time-evolution equation for rod-coil system under the effect of external fields. When applying a field to microphase separated rod-rich ellipsoidal domains, the domains rotate and merge to form string-like domains along the field direction which remain stable even after switching the field off. They also found that the magnitude of the field needed to rotate the domains depends on the rod to coil ratio.

Computational studies on the effects of external fields on nematogenic fluids have been presented by several groups. Monte Carlo simulations of Gay-Berne particles by Berardi et $a l .{ }^{30}$ illustrated that an electric field softens the isotropicsmectic transition by inducing a para-nematic weakly aligned phase in a system devoid of a zero-field nematic phase. Simulations by De Miguel et al. ${ }^{31}$ showed that a strong electric field promotes phases with positional order and increases the temperature range of the smectic phase, indicative of a coupling between directional and spatial order, with the smecticnematic transition becoming continuous beyond a critical field strength. Similarly, Luckhurst and Saillei ${ }^{32}$ observed a field-induced smectic-A phase from a nematic phase by Monte Carlo simulations of Gay-Berne mesogens, and interpreted this inducement as a coupling between rotational and translational order which shifts the boundary of the smectic-A phase to higher temperatures. Stimson and Wilson ${ }^{33}$ showed that a model side-chain liquid-crystal polymer, which shows microphase separation in zero field, can be made to order into a smectic-A layered structure by applying an aligning field. A field-induced nematic-smectic A transition was observed $^{34}$ in Monte Carlo simulations of bent-rod mesogens. Rotunno et al. $^{35}$ reported that short spherocylinders - too short to sustain ordered phases - can be ordered into polar nematic and hexagonal phases by an external field. The dissipative particle dynamics (DPD) method was used by Levine et $a l .{ }^{36,37}$ to study the re-alignment under a magnetic field of nematic and smectic nanodroplets suspended in an isotropic fluid.

Since practical nematogens often contain rigid and flexible sections, ${ }^{38}$ we here investigate by computer simulations the effect of external aligning fields on coarse-grained models of polymer liquid-crystal molecules. The simulations are performed using the DPD method, ${ }^{39-41}$ which has proven to be a powerful method to simulate complex fluids such as colloidal suspensions $s^{40,42}$ and block copolymers ${ }^{43,44}$ at mesoscopic time and length scales. We have recently applied DPD to study the liquid-crystalline properties of a fluid of rods and melts of rod-coil block copolymers. ${ }^{45,46}$ The fluid of rods, showing the isotropic, nematic, and smectic-A phases typical of liquid-crystalline ordering, serves as the reference system in the current study. Here, the rods will be adorned with one or two flexible tails and exposed to directional fields to study the resulting rich phase behavior of polymer liquid crystals, which find many practical applications as key components in flat panel displays, light shutters, and optical switches, among others. Evidently, the conformational freedom of the coils and the presence of interactions between rods and coils further complicate the phase behavior of polymer liquid-crystals by enabling microphase separation into a range of spatially ordered structures, while an alignment field provides an experimentalist or plant operator with an instrument to steer the resulting overall ordering process in a desired direction. Hence, the thermodynamically stable states of polymer liquid crystals arise through an intriguing interplay of several ordering mechanisms, in collaboration or in competition with each other, which is explored in this study. The simulations with conceptually simple coarse grained models contribute toward a qualitative understanding of the influences of the various ordering mechanisms on the emerging ordered phases of rod-coil block copolymer melts. The employed models, representing different polymer compositions, and the imposed field were specifically chosen to enable identifications of the dominant ordering mechanisms under a range of conditions, and to establish how the primary ordering mechanism can enhance a secondary ordering mechanism. 


\section{SIMULATION METHOD}

We used the DPD method ${ }^{39-41}$ to simulate the phase ordering of model liquid-crystalline polymers in external fields. Here, we give a summary of the method; further details can be found in our previous papers. ${ }^{45,46}$ Every particle in DPD represents a collection of atoms, just like the beads in a beadspring model of a polymer. The net force applied on particle $i$ is a sum of three pairwise additive forces,

$$
\mathbf{f}_{i}=\sum_{j \neq i} \mathbf{f}_{i j}^{C}+\sum_{j \neq i} \mathbf{f}_{i j}^{D}+\sum_{j \neq i} \mathbf{f}_{i j}^{R},
$$

where the sums run over all neighboring particles $j$ within a cut-off distance $r_{\mathrm{c}}$. These forces are a non-bonded conservative force $\mathbf{f}_{i j}^{C}=a_{i j} \omega_{C}\left(r_{i j}\right) \hat{\mathbf{r}}_{i j}$, a dissipative or friction force $\mathbf{f}_{i j}^{D}=-\gamma \omega_{D}\left(r_{i j}\right)\left(\hat{\mathbf{r}}_{i j} \cdot \mathbf{v}_{i j}\right) \hat{\mathbf{r}}_{i j}$, and a random or stochastic force $\mathbf{f}_{i j}^{R}=\sigma \omega_{R}\left(r_{i j}\right) \theta_{i j}(t) \hat{\mathbf{r}}_{i j}$. Here, the vector connecting the two particles is expressed as $r_{i j} \hat{\mathbf{r}}_{i j}=\mathbf{r}_{i}-\mathbf{r}_{j}$ with the hat denoting a unit vector. The strength and profile of the conservative force are set by $a_{i j}$ and the traditional choice $\omega_{C}\left(r_{i j}\right)=1-r_{i j} / r_{c}$, respectively. The Markovian random force, where $\theta_{i j}(t)$ is a time-dependent random number with zero average and unit variance, is related to the friction force through the fluctuation-dissipation theorem, which in the current case reads as $\sigma^{2} \omega_{R}^{2}(r)=2 \gamma \omega_{D}(r) k_{B} T$. Making the traditional choice $\omega_{D}=\omega_{R}^{2}=(1-r)^{2}$, one readily sees that the friction coefficient $\gamma$ and the fluctuation strength $\sigma$ set the temperature $T$ of the DPD thermostat, with $k_{B}$ being the Boltzmann constant. The dynamics of the DPD particles are calculated by numerically integrating Newton's equations of motion, for the above forces, by means of the Verlet leap-frog algorithm. ${ }^{47,48}$ In this paper, we follow the conventions of rescaled dimensionless parameters. The mass of the particles and the cut-off radius are chosen as the units of mass and distance, respectively, and the temperature (henceforth, we will use "temperature" to refer to $k_{B} T$ ) is expressed in terms of the unit of energy. The dimensionless time step has a value of $\delta t$ $=0.04$.

All liquid-crystal segments were modeled as rigid rods of seven fused spheres at fixed inter-particle distances of $r_{0}=2 / 3$. The resulting nematogen had a length to width ratio of about 5; we established that shorting these rods by one particle eradicated their liquid-crystalline phase behavior under the simulation conditions described below. During a simulation step, the two particles at the ends of a rod were moved under weighted averages of all forces acting on the seven rod particles, in combination with a constraint force supplied by the standard SHAKE routine to maintain a constant end-toend distance. ${ }^{47,49}$ At the end of the step, the positions of the five intermediate particles were readily calculated by a linear interpolation between the two end particles, thus creating a rigid rod. The perfectly rigid rods created by these constraints are devoid of the high frequency modes that arise when stiff bonds are used to generate rod-like segments, ${ }^{36,37}$ and therefore allow a larger time step. The suitability of the employed time step was confirmed by monitoring that both the kinetic and configurational temperatures of the fluids of rods, where the latter temperature was calculated using the approach outlined in Ref. 48 , stayed within $\sim 2 \%$ of the requested temper- ature over the studied temperature range, i.e., well within the limits set by Groot and Warren. ${ }^{41}$ Flexible coils were modeled as chains of several DPD particles connected by harmonic springs between adjacent beads, $\mathbf{f}_{i j}^{s}=C\left(r_{i j}-r_{0}\right) \hat{\mathbf{r}}_{i j}$, with the spring constant set at $C=4$ to obtain an average distance between adjacent beads comparable to that in the rods. This coarse-grained model of polymer liquid crystals is clearly not intended to quantitatively reproduce the phase behavior of any particular nematogen, but to provide a qualitative picture of the ordering processes in block copolymers based on their global compositions and to enable a comparison between various compositions.

The directional field was inspired by the simplest interaction of a neutral molecule in an external field, i.e., a dipole in a homogeneous field. In the simulations, this interaction is modeled by coupling the unit vector $\hat{\mathbf{u}}_{i}$ along the axis of the $i$ th rod-segment to an external field of strength $\phi$ pointing along the $z$-axis through the interaction potential

$$
\Phi_{i}=-\phi \hat{u}_{i z} .
$$

The net effect of this field was to apply a torque that rotated the rods toward alignment with the positive $z$-axis, while leaving their centers of mass unaffected. The coils were unaffected by the field. From the simulations discussed below it appears that field strengths below $\sim 10$ have relatively little effect on the ordering of the mesogens, while field strengths above $\sim 10$ can induce significant changes. Therefore, field strengths below 10 are referred to as "weak," while $\phi>10$ qualifies as "strong." All these intra-molecular conservative and constraint forces, as well as the effective forces resulting from the external field, were superimposed on the non-bonded forces of Eq. (1).

The simulations were carried out at constant pressure and temperature (NPT ensemble) using the Berendsen barostat ${ }^{47}$ in combination with DPD's innate thermostat. In order to establish an equilibrium isotropic pressure with a dimensionless value of 30 , while imposing as little restraints as possible on the formation of ordered phases, all six unique components of the set of box vectors were rescaled independently by the barostat. The desired temperature was obtained by varying the strength $\sigma$ of the fluctuating forces and keeping the friction force parameter constant at $\gamma=2.66$. This particular choice has the physically appearing advantage of making the variances of both forces, $\left\langle\left(\mathbf{f}_{i j}^{D}\right)^{2}\right\rangle$ and $\left\langle\left(\mathbf{f}_{i j}^{R}\right)^{2}\right\rangle$, scale linearly with temperature.

Extensive simulations were carried out to investigate the induction of liquid-crystalline phases in fluids of rods, diblock, and triblock copolymers under aligning fields of varying strengths. The degree of rotational order in these systems, and the increase thereof induced by the field, were analyzed quantitatively using the orientation order parameter $S_{2}$, which is defined as the largest positive eigenvalue of the ordering tensor

$$
\mathbf{Q}=\frac{1}{2}\left(3\left\langle\hat{\mathbf{u}}_{i} \hat{\mathbf{u}}_{i}\right\rangle-\mathbf{1}\right) .
$$

The numerical constants, with $\mathbf{1}$ being the unit matrix, set the range of the order parameter, which in this case runs from 0 for a rotationally disordered system to 1 for a perfectly aligned state. The average orientation of the rods is provided 
by the director, i.e., the eigenvector of $\mathbf{Q}$ with eigenvalue $S_{2}$. The possibility of field-induced orientational ordering resulting in positional ordering is also investigated, using distribution functions and visual inspection of the simulated systems. The degree of micro-phase separation in block copolymer systems is quantized by $Q_{1}=\left(\lambda_{2}+\lambda_{3}\right) / \lambda_{1}-2$, where $\lambda_{1} \leq \lambda_{2}$ $\leq \lambda_{3}$ are the three eigenvalues of the matrix $\mathbf{M}=\sum_{\mathbf{k}} \mathbf{k k} S(\mathbf{k})$ of second moments of the structure factors,

$$
S(\mathbf{k})=\frac{1}{N_{R}}\left\langle\left|\sum_{j=1}^{N_{R}} \exp \left(i \mathbf{k} \cdot \mathbf{r}_{j}\right)\right|^{2}\right\rangle,
$$

where the sums run over all $N_{R}$ rod particles. The resulting order parameter $Q_{1}$ is zero for isotropic structures and nonzero for microphase separated structures. ${ }^{45}$

\section{RESULTS AND DISCUSSION}

The first system to be discussed is a fluid of rods, which will serve as a reference. The simulations started with 3600 aligned rods, ordered in a crystalline state with 6 layers stacked along the $z$-direction, in a rectangular box measuring $14 \times 16 \times 28$, with the rods pointing along the field direction. The repulsion parameter between rods was set to $a_{R R}=20$. After equilibration at $T=0.1$, the temperature of this system was raised in a series of small steps. This procedure was applied in the absence of a field, and subsequently repeated for a number of increasingly stronger fields. Figure 1 shows the dependence of $S_{2}$ on temperature for five field strengths. In the absence of a field, the crystalline state was stable till a temperature of $\sim 0.3$ is reached. ${ }^{46} \mathrm{Next}$, an aligned and neatly stacked, but laterally disordered, smectic phase was stable upto $T \approx 0.44$. At this temperature, the systems transformed into a positionally disordered, but still well-aligned with $S_{2}=0.87$, nematic phase. The orientational order steadily decreased with increasing temperature to $S_{2}=0.67$ at $T \approx 0.88$. Beyond the clearing (nematic-

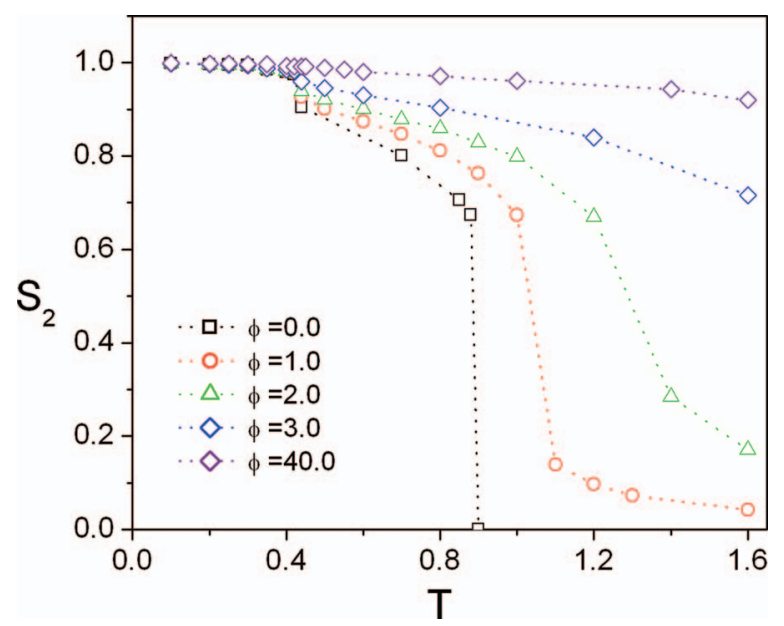

FIG. 1. The orientational order parameter $S_{2}$ as a function of temperature for a fluid of rods, under external alignment fields of various strengths $\phi$. From left to right, the zero-field system transforms from crystalline to smectic at $T \approx 0.3$, to nematic at $T \approx 0.44$, and finally to isotropic at $T \approx 0.88$. The discontinuities at the transitions become smaller, and eventually disappear, with increasing field strength. isotropic) point at $T_{N I} \approx 0.9$ the ordering completely vanished, with $S_{2}$ dropping to a thermal noise level close to zero, in a sharp nematic-isotropic transition. Since the pressure was kept constant, the density of the system made small jumps $(\Delta \rho<1 \%)$ at the transition points and changed smoothly between the transitions. ${ }^{45}$ In comparison with experimental nematogens such as $8 \mathrm{CB}$, the nematic phase is much wider. This is a consequence of the employed highly idealized model of straight rods interacting only by a soft repulsive potential. Interactions between experimental mesogens often include various attractive contributions, such as Van der Waals attractions, dipole-dipole interactions, and $\pi-\pi$ stacking, besides strong excluded volume interactions. All of these interactions affect the phase behavior, and consequently a quantitative difference with the current simulations is both expected and acceptable.

Exposing the isotropic phase to a weak field, $\phi=1$, induced a weak alignment of the rods, known as a para-nematic phase. In the nematic phase, the order also increased relative to the zero-field state at the same temperature. The field raised the clearing point by about 0.15 , while the jump in $S_{2}$ at the transition became smaller. For $\phi=2$ the jump decreased further, as the ordering of the para-nematic state steadily increased with the field strength while the nematic state bordering the clearing point remained at $S_{2} \sim 0.67$. The discontinuity at the phase transition diminished with the increasing field strength, and is estimated from the simulations to vanish at a critical field strength of about 2.3 and a critical temperature of $\sim 1.4$. A detailed characterization of this criticality, which by theory and experiments has been identified as a liquid-gas-like critical point, ${ }^{4,9,17}$ lies outside the scope of the current study. In stronger fields a continuous change-over resulted between nematic and para-nematic states.

Critical points of istropic-nematic transitions have been measured for a few nematogens. Lelidis and Durand ${ }^{3}$ report for $5 \mathrm{CB}$ a critical electric field of $E_{\mathrm{c}}=140 \mathrm{kV} / \mathrm{cm}$ at a critical temperature of $T_{\mathrm{c}}=307.5 \mathrm{~K}$. These data were confirmed by Basappa and Madhusudana, ${ }^{2}$ who for $80 \mathrm{CB}$ found a less clear critical point at $E_{\mathrm{c}} \approx 160 \mathrm{kV} / \mathrm{cm}$ and $T_{\mathrm{c}} \approx 353 \mathrm{~K}$. An order of magnitude calculation then shows that the energy change upon reverting the direction of a mesogen from parallel to antiparallel to the electric field, $\Delta E=2 p \varepsilon_{\mathrm{r}} E_{\mathrm{c}}$ with $p \approx 4-5 \mathrm{D}$ being the dipole moment and $\varepsilon_{\mathrm{r}} \approx 10$ being the dielectric constant, respectively ${ }^{50-52}$ is roughly equal to the thermal energy, $\Delta E / k_{\mathrm{B}} T_{\mathrm{c}} \approx 3-4$, in the experimental systems at their critical temperature. A similar estimate based on the dimensionless values of the simulated system yields a scaled energy change for reverting a nematogen of $2 \phi_{\mathrm{c}} / T_{\mathrm{c}} \approx 3$. The agreement between these two scaled ratios is better than anticipated for this simple coarse-grained model.

Weak fields hardly improved the ordering of the smectic phase, and the temperatures of the crystal-smectic and smectic-nematic transitions did not appear to shift significantly. The step of $S_{2}$ at the latter transition decreased with the increasing field strength. A field-induced shift of the smecticnematic transition is better studied by looking at the positional order. Figure 2 shows distributions $g_{c m}(z)$ of the rods' centers of mass along the direction of the aligning field, which is also the average orientation of the director. The plots in the absence of a field, see Fig. 2(a), show the sharp peaks of the 


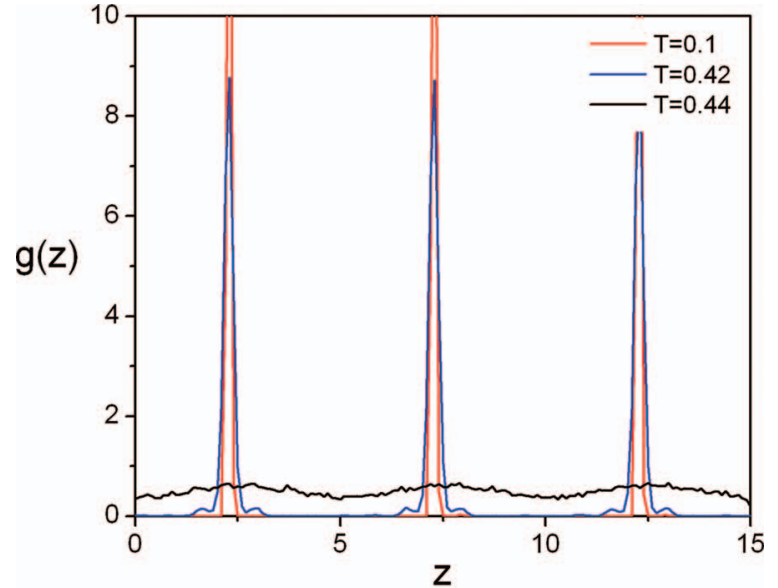

(a)

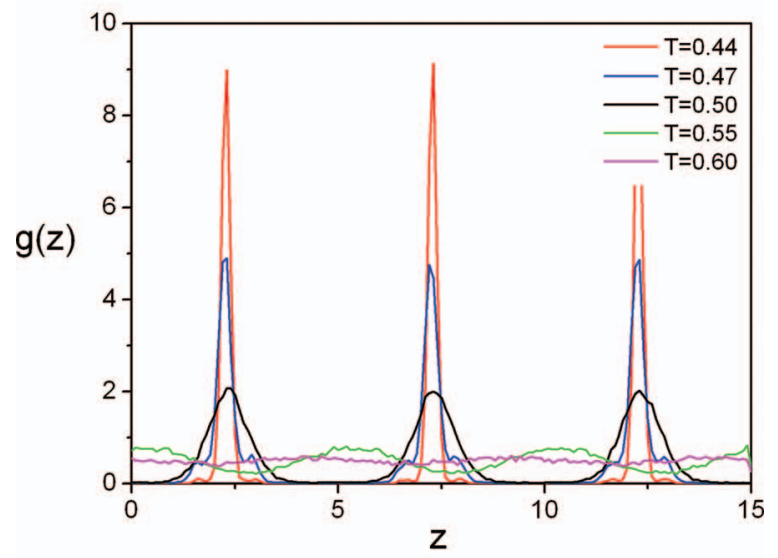

(b)

FIG. 2. The distributions $g_{c m}(z)$ of the rods' centers of mass along the direction of the external field for the fluid of rods (a) in zero-field show a sharp phase transition with temperature from smectic to nematic, which turns into a gradual transition (b) over a temperature range under a strong alignment field, $\phi=40$. Two snapshots of the latter transition are shown in Fig. 3. Both the time-averaged long axis of the simulation box and the time-averaged director are parallel to the field.

highly ordered crystalline phase at $T=0.1$ and the slightly broader peaks of a smectic phase at $T=0.42$. The latter acquired small secondary peaks of rods that are slightly sticking out of their respective layers. The peaks vanish for $T=0.44$, as expected for a nematic phase. For weak fields, $\phi<10$, the distribution curves are almost identical to the ones shown here, thus confirming that these fields do not induce appreciable shifts of the phase boundaries between the three ordered liquid crystalline phases. For strong fields, such as $\phi=40$, the high value of $S_{2}$ for all temperatures considered, see Fig. 1, indicates that the rods are always well aligned. Unlike the weak-field systems, there is no sharp jump around $T$ $=0.44$, which suggests that the smectic-nematic transition has also become continuous. This conclusion is supported by the distribution of the rods' centers of mass in Fig. 2(b): the sharp peaks in $g_{c m}(z)$ at $T=0.44$ do not suddenly disappear upon a small temperature increment, but instead gradually broaden and decrease in intensity as the temperature increases to 0.55 , and the remaining undulations eventually vanish to a homogeneous distribution at $T=0.60$. Figure 3 shows snapshots of the simulation boxes at $T=0.50$ and 0.55 . While the

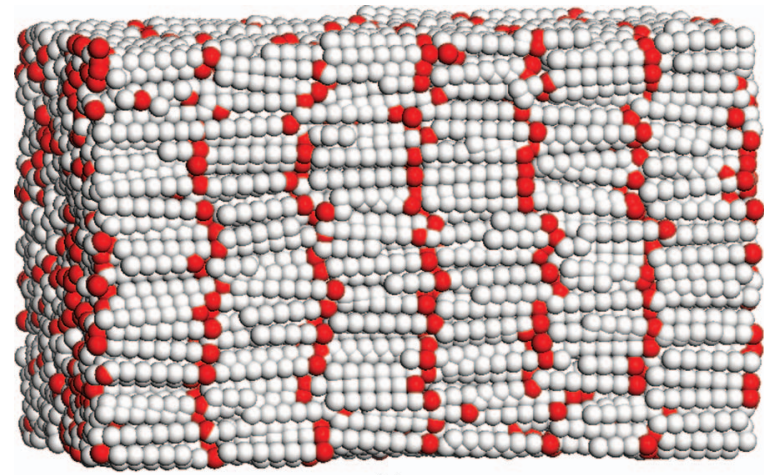

(a)

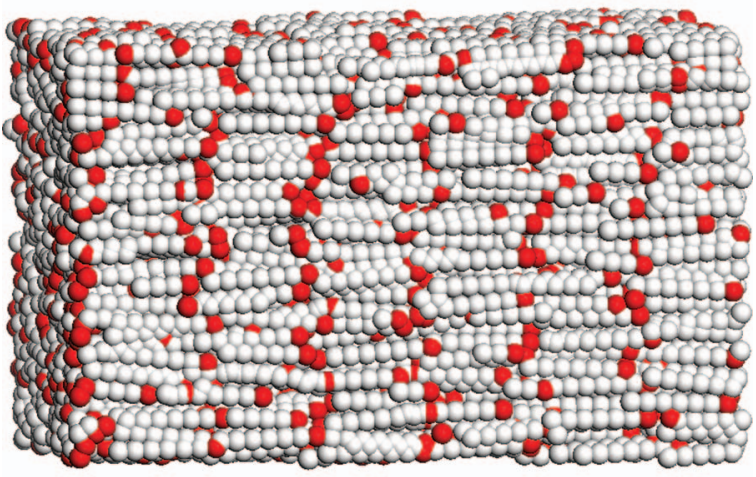

(b)

FIG. 3. Snapshots of the fluid of rods in the continuous smectic to nematic transition under a strong alignment field, $\phi=40$. The system changes from (a) neatly stacked at a temperature of 0.50 to (b) small domains at $T=0.55$ to an aligned structure-less state at $T=0.60$ (not shown). For clarity, the first particle of every rod is colored red. The applied field points to the right.

rods are nicely stacked at the lower temperature, the interfaces between the layers are less ordered at the higher temperature. At $T=0.55$ the system appears to consist of a number of ordered domains, separated from their neighbors by less ordered boundaries; this fragmented ordering did not disappear by prolonging the simulations. Note that the strong field induces sharp peaks in $g_{c m}(z)$ at $T=0.44$, where the system in the weak-field does not support a smectic phase at this temperature. Additional simulations were carried out to estimate the value of the critical point at which the first order smecticnematic transition disappears. The discontinuity in the value of $S_{2}$ vanishes completely at the critical field $\phi_{\mathrm{c}} \approx 25$ and critical temperature $T_{\mathrm{c}} \approx 0.45$. This field strength can be compared to the critical electric field of $E_{\mathrm{c}}=350 \mathrm{kV} / \mathrm{cm}$ at $T_{\mathrm{c}} \approx 306 \mathrm{~K}$ reported for the non-spontaneous-nematicsmectic A transition in experiments on an 8CB-10CB mixture $^{5}$ and to the $E_{\mathrm{c}} \approx 15 \mathrm{~V} / \mathrm{cm}$ at $T_{\mathrm{c}} \approx 306 \mathrm{~K}$ for a nematic-smectic A transition in pure $8 \mathrm{CB} .{ }^{19}$ Repeating the above order of magnitude calculation then shows that the energy change upon reverting the direction of a mesogen from parallel to antiparallel to the electric field, with $p \approx 5 \mathrm{D}$ being the dipole moment and $\varepsilon_{\mathrm{r}} \approx 10$ being the dielectric constant of $8 \mathrm{CB}$, respectively, ${ }^{52}$ yields $\Delta E / k_{\mathrm{B}} T_{\mathrm{c}}=5-10$. The estimate based on the dimensionless values of the simulated system yields a scaled energy change for reverting a nematogen of $2 \phi_{\mathrm{c}} / T_{\mathrm{c}} \approx 10^{2}$. The agreement between these two scaled ratios is reasonable, considering the previously discussed differences between the simplified interactions in the highly 


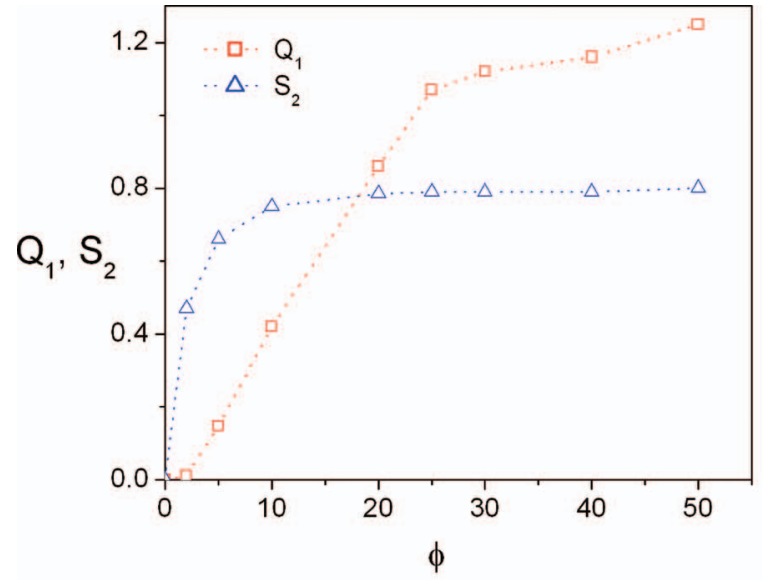

(a)

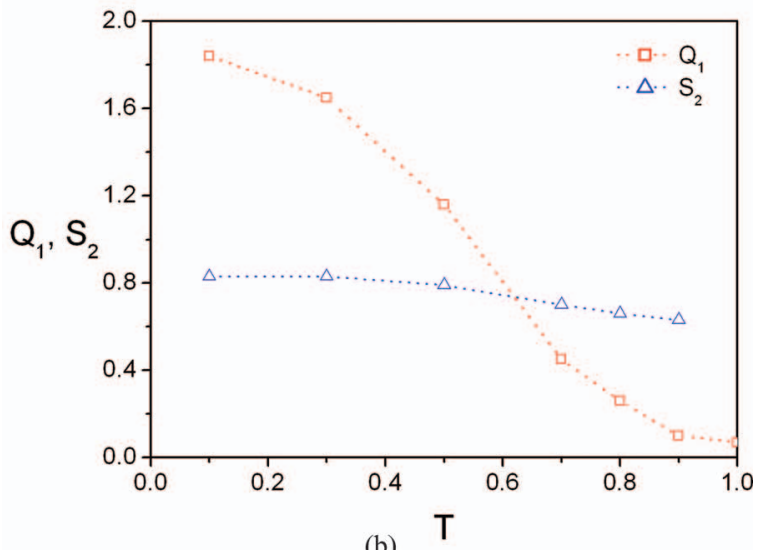

(b)

FIG. 4. Micro-phase separation parameter $Q_{1}$ and orientation order parameter $S_{2}$ of the athermal, $\Delta a=0$, diblock copolymer $\mathrm{R}_{7} \mathrm{C}_{7}$ system plotted (a) as functions of the field strength $\phi$ at $T=0.5$ and (b) as functions of the temperature for a strong field, $\phi=40$. The alignment of the rods by an external field induces translational ordering. A snapshot of this system is shown in Fig. 5.

idealized simulation model, the multitude of interactions between experimental nematogens, and the approximate nature of this order-of-magnitude estimate. We conclude that strong fields shift the phase-change to higher temperatures, and even make the change-over a continuous one for $\phi \geq 25$. A detailed characterization of the nature of this phase point, which is predicted to be a tricritical point, ${ }^{13}$ exceeds the scope of this study.

The second simulated system contained 3000 diblock copolymers $\left(\mathrm{C}_{7} \mathrm{R}_{7}\right)$, in which the rigid rod of 7 beads is adorned with a flexible coil also of 7 beads, with all coilcoil, rod-rod, and rod-coil interaction strengths set at the same value, $a_{C C}=a_{R R}=a_{R C}=20\left(\Delta a=a_{R C}-a_{j j}=0\right)$. The copolymers were placed at random in a cubic box of size $22 \times 22 \times 22$ and equilibrated in an NPT simulation at a high temperature, before being quenched to a desired temperature in the equilibration runs preceding the production runs. Figure 4(a) shows the directional and micro-phase order parameters as a function of field strength, for $T=0.5$. In the absence of a field, and despite the low temperature which sees the fluid of rods order in a nematic phase, the rod-like segments of the block copolymers have neither aligned nor formed layers, indicating that the attached coils suppress the rods' nematogenic properties. Applying a fairly weak field of

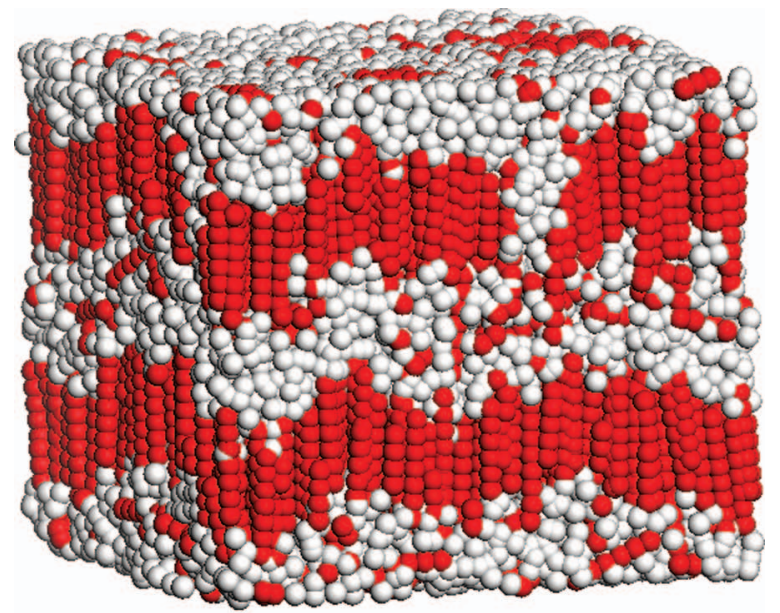

(a)

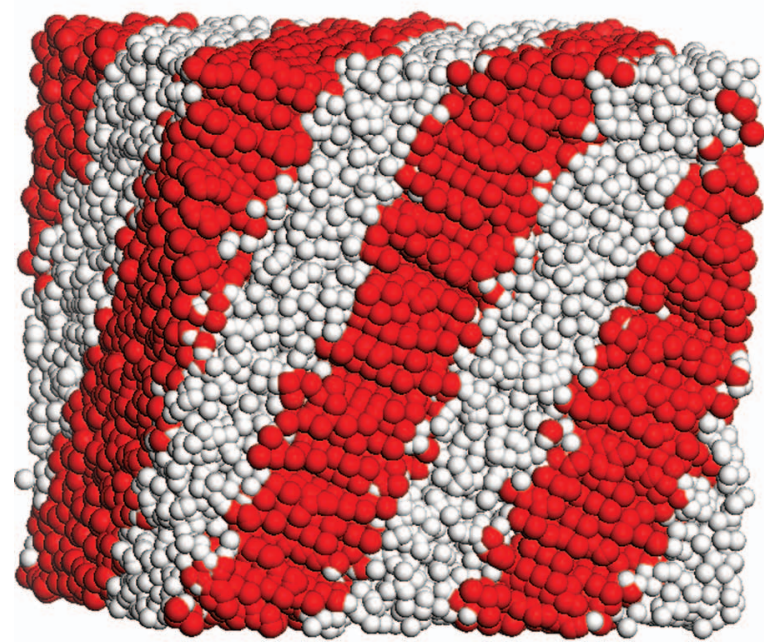

(b)

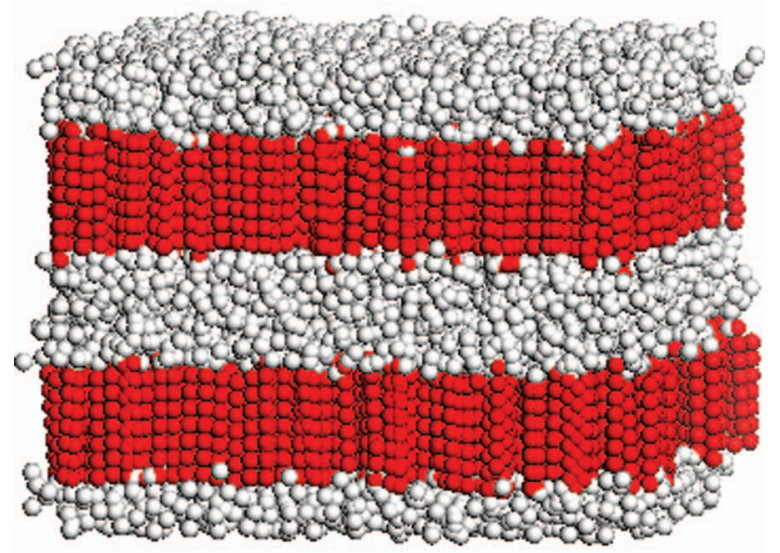

(c)

FIG. 5. The diblock copolymer $\mathrm{R}_{7} \mathrm{C}_{7}$ system with commensurate rod (red) and polymer (grey) particles, $\Delta a=0$, does not order at $T=0.3$ in the absence of a field, see Fig. 4, but forms irregular smectic-like layers (a) in a strong field, $\phi=40$. For incommensurate rod and coil particles, $\Delta a=5$, the $\mathrm{R}_{7} \mathrm{C}_{7}$ system at $T=0.3$ micro-phase separates into regular lamellae (b) perpendicular to the $\phi=40$ field or in a random direction (c) in the absence of a field.

$\phi=2$, however, suffices to partially align the rigid segments, and the ordering saturates at an intermediate field strength of $\phi=10$. Interestingly, once the nematogens are sufficiently aligned, at $S_{2} \approx 0.7$, the systems start to micro-phase 


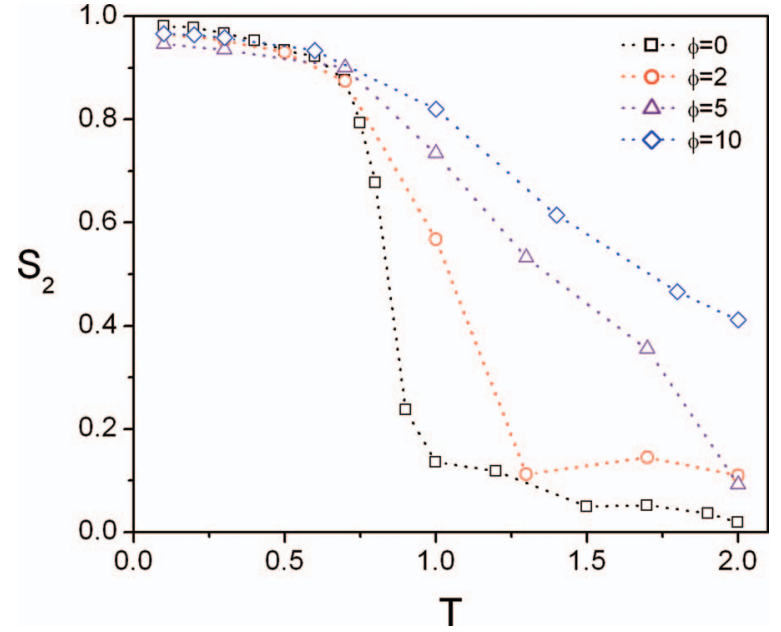

FIG. 6. The orientational order parameter $S_{2}$ for diblock copolymers $\mathrm{R}_{7} \mathrm{C}_{7}$ as a function of temperature for various field strengths. The incommensurability of the rod and coil particles, $\Delta a=5$, makes the loss of orientational order with increasing temperature a smoother transition than for the fluid of rods, see Fig. 1. An alignment field further smoothens and broadens the transition. The noise on the data reflects the slow assembly of uniform lamellar phases when quenching disordered systems from high $\mathrm{T}$ and $\Delta a=0$ to the desired $T$ and $\Delta a=5$; the system at $\phi=2$ and $T=1.3$ became trapped in a fragmented final state.

separate as well. The snapshot in Fig. 5(a) shows that the resulting layers are strongly undulating, even in a strong field of $\phi=40$, suggesting that the mechanism driving the segregation is fairly weak. In this strong field, the micro-phase and orientational ordering steadily decrease with increasing temperature, see Fig. 4(b). The observation that $Q_{1}$ drops to zero well before $S_{2}$ once more suggests that in this symmetric polymer-rod system positional ordering emerges as a consequence of the induced alignment.

Microphase separation of block copolymers occurs when the rod and coil particles do not mix spontaneously. To observe this effect, we repeated the above simulations from random start configurations with the repulsion parameter between unlike particles increased to $a_{R C}=25$, while those for like particles remained at $a_{i i}=20$. Figure 5(b) shows that this $\Delta a=a_{R C}-a_{i i}=5$ system forms a well defined lamellar structure at $T=0.5$, even in zero-field, unlike the $\Delta a=0$ system. The degree of ordering below $T=0.7$ hardly improves upon applying a field, as quantified in Fig. 6, but the snapshots in Figure 5(c) reveal that the orientation of the lamellae and director is clearly affected when a field is applied. In zero-field, the order parameter makes a major jump around a temperature of 0.8 , marking the transition between lamellar phases with and without closely aligned rods. The proximity of this transition temperature to the clearing point of the fluid of rods is probably not accidental. The residual $S_{2}$ for $T>0.8$ reflects the surviving ordering of the rods in layers. Since the rods of end-to-end length 4 cross a layer of thickness $d$ from one interface to the next, it follows that $\hat{u}_{i z} \approx d / 4$ and hence that $S_{2}$ is a simple function of $d$. Applying a field promotes orientational order and therefore smoothes the transition and shifts it to higher temperatures. With increasing temperature the layers become less well-defined and consequently $S_{2}$ de-

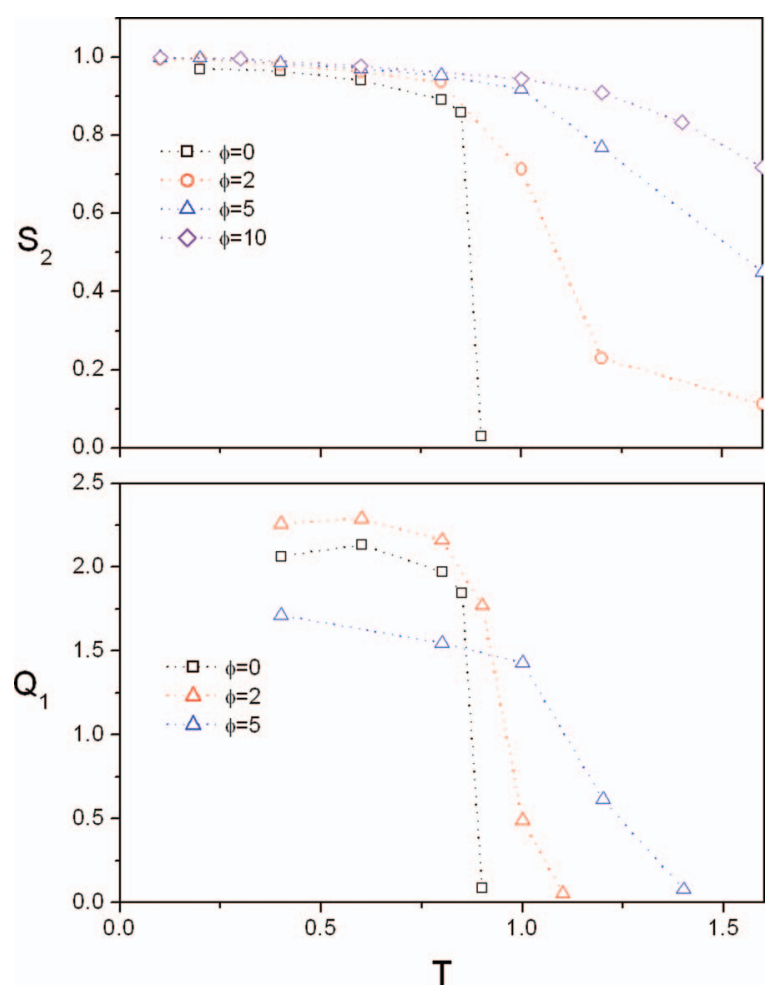

FIG. 7. Ordering of triblock copolymers $\mathrm{C}_{3} \mathrm{R}_{7} \mathrm{C}_{3}$, with $\Delta a=5$, as a function of temperature for various field strengths, revealing close coupling between the loss of orientational order $S_{2}$ and the loss of translational order $Q_{1}$ with increasing temperature. The disordering transition of the triblock, especially at zero field, is markedly different from that of the $\mathrm{R}_{7} \mathrm{C}_{7}$ diblock, see Fig. 6 , despite containing (almost) the same numbers of rod and chain particles.

creases, until the micro-phase separation ceases and the system becomes isotropic.

Another symmetric block copolymer, of similar rod-tocoil ratio $f$ as the above diblock, is obtained by adorning both ends of the rod with a short flexible chain, as in the triblock $\mathrm{C}_{3} \mathrm{R}_{7} \mathrm{C}_{3}$. Simulations were again initiated by placing 3000 copolymers at random in a cubic $22 \times 22 \times 22$ box, followed by equilibration under NPT conditions at an elevated temperature and $\Delta a=0$. These homogeneous systems were then quenched to the desired temperature and $\Delta a=5$ to produce the ordering curves presented in Fig. 7. A marked difference with the equal length diblock copolymers is the much sharper order-disorder transition (ODT) around $T \approx 0.85$ in the absence of a field. The transition is reminiscent of the nematic-isotropic transition of the fluid of rods (see Fig. 1) and unlike the residual order observed for the diblocks (see Fig. 6), in that both order parameters suddenly vanish at the ODT. Combined, this strongly suggests that the triblocks' ordered phase is stabilized by nematogenic behavior with a supportive role by the repulsions between rod and coil particles (which enhances $S_{2}$ relative to the fluid of rods, but hardly shifts the clearing transition), as opposed to the diblocks' ordered phase which is stabilized by micro-phase separation of the rods and coils and enhanced by nematogenic ordering. Applying an alignment field reduces the jumps of $S_{2}$ and $Q_{1}$ at the transition and shifts the transition to higher temperatures, as for the fluid of rods. Interestingly, in non-zero fields the loss of rotational ordering occurs over a considerably wider 
temperature range than the loss of positional ordering. This confirms the notion that para-nematic ordering, when sufficiently high, induces domain formation for these triblocks.

Finally, we studied the effect of an alignment field on diblock copolymer systems whose low rod-to-coil ratio renders them incapable of micro-phase separation into lamellar phases. We have carried out simulations of a homogeneous melt of $2000 \mathrm{R}_{7} \mathrm{C}_{28}(f=0.20)$ diblock copolymers initially

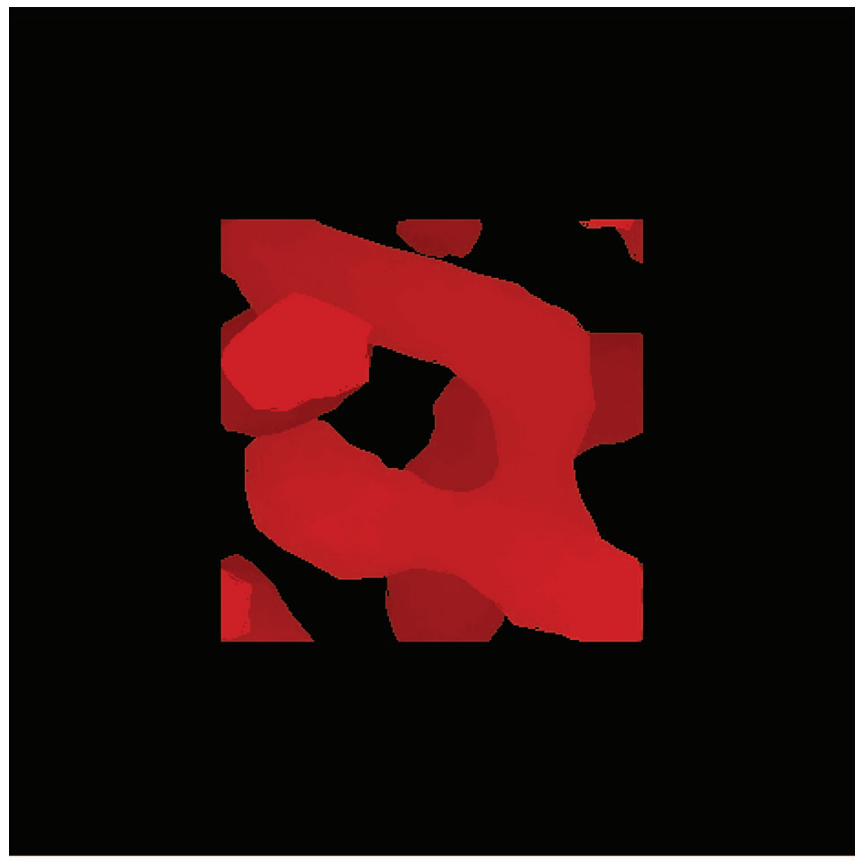

(a)

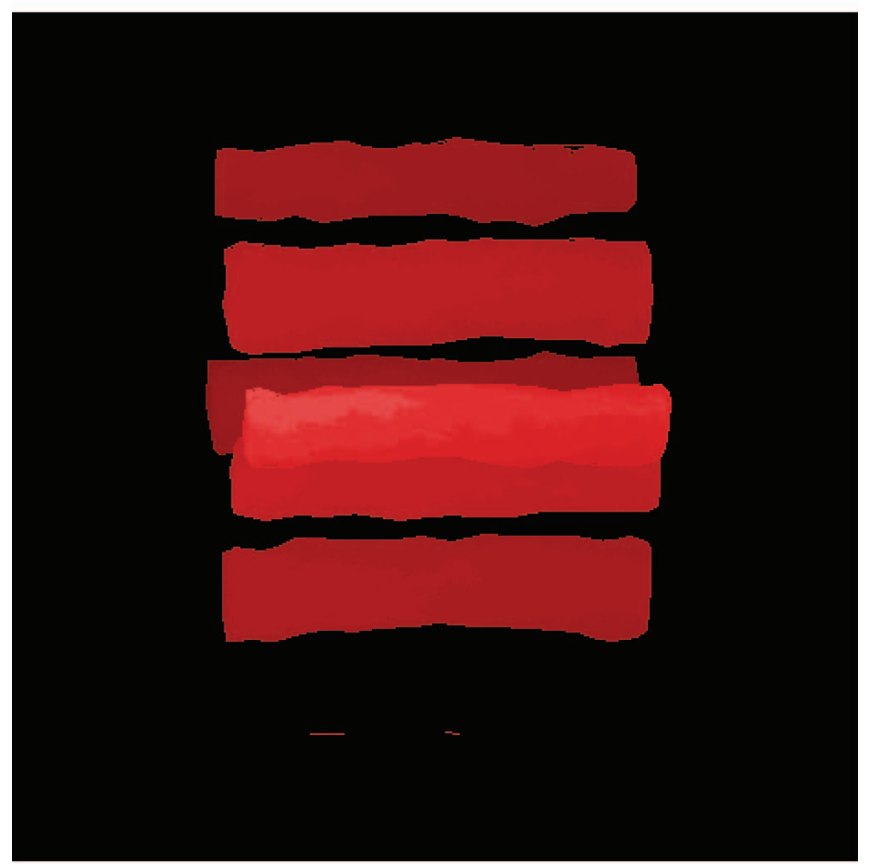

(b)

FIG. 8. Snapshots of micro-phase separated $\mathrm{R}_{7} \mathrm{C}_{28}(f=0.20)$ diblock copolymers, for $T=1.0$ and $\Delta a=15$, showing only the rods. The system forms (a) bicontinuous phases with no preferential direction in zero-field, but parallel cylindrical microphases of rods in a weak field $\phi=5$ directed along the horizontal direction. placed in a $26 \times 26 \times 26$ box, at $T=1.0$ and $\Delta a=15$. This system ordered in zero-field into a bi-continuous phase devoid of orientational order, see Fig. 8(a). When the simulation was repeated in a weak field, $\phi=5$, the rods formed cylindrical micelles parallel to the field direction, as illustrated in Fig. 8(b). The even more asymmetric diblock copolymer $\mathrm{R}_{7} \mathrm{C}_{43}(f=0.14)$ was observed to form spherical micelles, containing the randomly oriented rods, in the absence of the field. The micelles became elliptical and started to display alignment ordering at $\phi=10$ (not shown). This alignment of domains is observed experimentally for rodcoil copolymers. ${ }^{24-27}$ Micro-phase morphology transformations under an external field were also observed in coil-coil copolymers. ${ }^{53-57}$ In particular, the self-consistent mean field calculations reported by Ly et al. ${ }^{53}$ showed a possible gyroidto-cylindrical micelles transformation of a diblock copolymer melt resulting from the application of an electric field. This behavior was confirmed experimentally by Schmidt et al. ${ }^{54}$ who showed that the application of a sufficiently strong electric field forced polystyrene-b-polyisoprene in toluene to undergo a phase transition from the gyroid to the cylindrical phase.

\section{CONCLUSIONS}

In this study, the effect of an aligning field on the orientational ordering and micro-phase separation of model polymer liquid crystals was investigated by mesoscale computer simulations using the dissipative particle dynamics method. The results on rod fluids show good agreement with previous simulations using Gay-Berne nematogens. ${ }^{30-32}$ In the presence of weak fields, the clearing point shifts to higher temperatures and a weakly ordered para-nematic phase is induced in the zero-field isotropic region. The discontinuity at the para-nematic-nematic transition softens and vanishes for sufficiently strong fields. A similar shift and softening are induced, for strong fields, in the otherwise discontinuous smectic-nematic transition. Note that in this case the stimulated high degree of orientational ordering brings about a transformation of the positional ordering. Molecular field theory and Monte Carlo simulations by Luckhurst and Saillei ${ }^{32}$ also predicted the stabilization of the smectic-A phase and the shift in the smectic-nematic transition temperature under an external field. The induced formation of smetic-A structure has also been reported for Gay-Berne particles, ${ }^{31}$ which in the absence of a field do not possess a stable smectic phase.

Field-induced orientational alignment also results in positional ordering in melts of rod-coil block copolymers. This is most clearly seen for athermal symmetric diblocks ( $\Delta a=0$ and $f=0.5$ ) that do not posses any ordering in zero field, but whose para-nematic ordering in a strong alignment field causes a transition to a "para-smectic" layered structure when the induced orientational order exceeds a critical value of about 0.7. Alignment fields also stabilize micro-phase separated layering of incompatible block copolymers by shifting the disordering transition to higher temperatures. This shifting in the transition temperature caused by the field was also observed experimentally. ${ }^{23}$ In highly asymmetric rodcoil block copolymers, the presence of an alignment field 
affects the morphology of the mesophases. As an example, the continuous network formed by the minority rod component in an $f=0.2$ diblock was made to change into a hexagonal array of cylinders. We conclude that coarse-grained DPD simulations provide a convenient computational tool to explore and understand the complex phase behaviour of polymer liquid crystals in an external field.

\section{ACKNOWLEDGMENTS}

Abdullah AlSunaidi would like to thank the King Fahd University of Petroleum and Minerals (KFUPM) for financial support.

${ }^{1}$ W. Helfrich, Phys. Rev. Lett. 24, 201 (1970).

${ }^{2}$ G. Basappa and N. Madhusudana, Mol. Cryst. Liq. Cryst. 288, 161 (1996).

${ }^{3}$ I. Lelidis and G. Durand, Phys. Rev. E 48, 3822-3824 (1993).

${ }^{4}$ E. I. Rjumtsev, M. A. Osipov, T. A. Rotinyan, and N. P. Yevlampieva, Liq. Cryst. 18, 87 (1995).

${ }^{5}$ I. Lelidis and G. Durand, Phys. Rev. Lett. 73, 672 (1994).

${ }^{6}$ C. Rosenblatt, Phys. Rev. A 24, 2236 (1981).

${ }^{7}$ T. Ostapenko, D. B. Wiant, S. N. Sprunt, A. Jakli, and J. T. Gleeson, Phys. Rev. Lett. 101, 247801 (2008).

${ }^{8}$ J. Tang and S. Fraden, Phys. Rev. Lett. 71, 3509 (1993).

${ }^{9}$ P. J. Wojtowicz and P. Sheng, Phys. Lett. A 48, 235 (1974).

${ }^{10}$ A. R. Khokhlov and A. N. Semenov, Macromolecules 15, 1272 (1982).

${ }^{11}$ H. H. Wensink and G. J. Vroege, Phys. Rev. E 72, 031708 (2005).

${ }^{12}$ C. Rosenblatt, Phys. Lett. A 83, 221 (1981); J. Phys. Lett. 42, 9 (1981).

${ }^{13}$ E. F. Gramsbergen, L. Longa, and W. H. de Jeu, Phys. Rep. 135, 195-257 (1986).

${ }^{14}$ I. Lelidis and G. Durand, J. Phys. II 6, 1359 (1996).

${ }^{15}$ H. Hama, J. Phys. Soc. Jpn. 54, 2204 (1985).

${ }^{16}$ M. Torikai and M. Yamashita, J. Phys. Soc. Jpn. 73, 2154 (2004).

${ }^{17}$ A. J. Nicastro and P. H. Keyes, Phys. Rev. A 30, 3156 (1984).

${ }^{18}$ S. Dhara and N. V. Madhusudana, Eur. Phys. J. E 22, 139-149 (2007).

${ }^{19}$ I. Lelidis, Phys. Rev. Lett. 86, 1267 (2001).

${ }^{20}$ A. Yethiraj, R. Mukhopadhyay, and J. Bechhoefer, Phys. Rev. E 65, 021702 (2002).

${ }^{21}$ A. Yethiraj, in Thermotropic Liquid Crystals: Recent Advances (Springer, 2007), p. 235.

${ }^{22}$ S. Singh, Phys. Rep. 324, 107-269 (2000).

${ }^{23}$ B. McCulloch, G. Portale, W. Bras, and R. A. Segalman, Macromolecules 44, 7503-7507 (2011).

${ }^{24}$ Y. Tao, H. Zohar, B. D. Olsen, and R. A. Segalman, Nano Lett. 7, 2742 (2007).

${ }^{25}$ M. Gopinadhan, P. W. Majewski, and C. O. Osuji, Macromolecules 43, 3286-3293 (2010).
${ }^{26}$ M. Gopinadhan, P. W. Majewski, Y. Choo, and C. O. Osuji, Phys. Rev. Lett. 110, 078301 (2013).

${ }^{27}$ I. W. Hamley, V. Castelletto, Z. B. Lu, C. T. Imrie, T. Itoh, and M. Al-Hussein, Macromolecules 37, 4798-4807 (2004).

${ }^{28}$ T. Ohta, N. Yamazaki, M. Motoyama, K. Yamada, and M. Nonomura, J. Phys.: Condens. Matter 17, S2833-S2840 (2005).

${ }^{29}$ N. Yamazaki, M. Motoyama, M. Nonomura, and T. Ohta, J. Chem. Phys. 120, 3949 (2004).

${ }^{30}$ R. Berardi, S. Orlandi, and C. Zannoni, Mol. Cryst. Liq. Cryst. 394, 141 (2003).

${ }^{31}$ E. De Miguel, F. J. Blas, and E. M. Del Rio, Mol. Phys. 104, 2919 (2006).

${ }^{32}$ G. R. Luckhurst and G. Saillei, J. Chem. Phys. 112, 4342 (2000).

${ }^{33}$ L. M. Stimson and M. R. Wilson, J. Chem. Phys. 123, 034908 (2005).

${ }^{34}$ J. Xu, R. Selinger, J. Selinger, B. Ratna, and R. Shashidhar, Phys. Rev. E 60, 5584 (1999).

${ }^{35}$ M. Rotunno, T. Bellini, Y. Lansac, and M. Glaser, J. Chem. Phys. 121, 5541 (2004).

${ }^{36}$ Y. K. Levine and A. Polimeno, Eur. Phys. J. E 23, 13-23 (2007).

${ }^{37}$ Y. K. Levine and W. Bras, Eur. Phys. J. E 25, 5-16 (2008).

${ }^{38}$ H. Kelker and R. Hatz, Handbook of Liquid Crystals (Chemie, Weinheim, Deerfield, 1980).

${ }^{39}$ P. J. Hoogerbrugge and J. M. V. A. Koelman, Europhys. Lett. 19, 155 (1992).

${ }^{40}$ J. M. V. A. Koelman and P. J. Hoogerbrugge, Europhys. Lett. 21, 363 (1993).

${ }^{41}$ R. D. Groot and P. B. Warren, J. Chem. Phys. 107, 4423 (1997).

${ }^{42}$ E. S. Boek, P. V. Coveney, H. N. W. Lekkerkerker, and P. van der Schoot, Phys. Rev. E 55(3), 3124-3133 (1997).

${ }^{43}$ R. D. Groot and T. J. Madden, J. Chem. Phys. 108, 8713 (1998).

${ }^{44}$ R. D. Groot, T. J. Madden, and D. J. Tildesley, J. Chem. Phys. 110, 9739 (1999).

${ }^{45}$ A. AlSunaidi, W. K. Den Otter, and J. H. R. Clarke, Philos. Trans. R. Soc. London, Ser. A 362, 1773 (2004).

${ }^{46}$ A. AlSunaidi, W. K. Den Otter, and J. H. R. Clarke, J. Chem. Phys. 130, 124910 (2009).

${ }^{47}$ M. P. Allen and D. J. Tildesley, Computer Simulation of Liquids (Clarendon, Oxford, 1987).

${ }^{48}$ W. K. den Otter and J. H. R. Clarke, Int. J. Mod. Phys. C 11, 1179 (2000).

${ }^{49}$ G. Ciccotti, M. Ferrario, and J. P. Ryckaert, Mol. Phys. 47, 1253 (1982).

${ }^{50}$ B. R. Ratna and R. Shashidhar, Pramana 6, 278 (1976).

${ }^{51}$ S. Czerkas, A. Burczyk, G. Czechowski, and J. Jadzyn, "Polymers and liquid crystals," Proc. SPIE 4017, 192 (1999).

${ }^{52}$ S. C. Bharath, K. R. Pimputkar, A. M. Pronschinske, and T. P. Pearl, Appl. Surf. Sci. 254, 2048 (2008).

${ }^{53}$ D. Q. Ly, T. Honda, T. Kawakatsu, and A. V. Zvelindovsky, Macromolecules 40, 2928 (2007).

${ }^{54}$ K. Schmidt, C. W. Pester, H. G. Schoberth, H. Zettl, K. A. Schindler, and A. Boker, Macromolecules 43, 4268 (2010).

${ }^{55}$ A. V. Kyrylyuk, A. V. Zvelindovsky, G. J. A. Sevink, and J. G. E. M. Fraaije, Macromolecules 35, 1473-1476 (2002).

${ }^{56}$ T. Xu, Y. Zhu, S. P. Gido, and T. P. Russell, Macromolecules 37, 2625-2629 (2004).

${ }^{57}$ T. Xu, A. V. Zvelindovsky, G. J. A. Sevink, O. Gang, B. Ocko, Y. Q. Zhu, S. P. Gido, and T. P. Russell, Macromolecules 37(18), 6980-6984 (2004). 